\title{
Chest computed tomography in COVID-19 infection
}

\author{
Filippo Crimì ${ }^{1}$ (I) - Giulio Cabrelle ${ }^{1} \cdot$ Chiara Zanon $^{1} \cdot$ Emilio Quaia $^{1}$
}

Received: 30 June 2021 / Accepted: 27 July 2021 / Published online: 5 August 2021

(c) Italian Association of Nuclear Medicine and Molecular Imaging 2021

\section{Dear Editor in Chief,}

We read with great interest the recently published metaanalysis by Pang et al. about chest CT as a primary tool in the detection of COVID -19 infection [1]. Several physicians employed chest high-resolution CT (HRCT) to confirm the diagnosis of COVID-19 or, especially at the beginning of the pandemic, even to substitute the real time-polymerase chain reaction (RT-PCR) because of the suboptimal accuracy of the first screening tests and of the paucity of reagents. The authors surely should be commended for the huge effort made in summarizing and analyzing the heterogeneous literature about this topic, involving different techniques, settings of care, and timing of studies with consequent different awareness about radiological signs and symptoms of this infection. The high sensitivity (94\%) and the excellent area under the receiver operating characteristic (ROC) curve (0.93) reported by Pang et al. confirm the role of chest CT as a fundamental diagnostic tool in COVID-19 infection. Nevertheless, some concerns could be raised, especially about the inclusion criteria adopted in this meta-analysis which, in our opinion, suggest to take with caution at least some of the conclusions drawn by the study.

First of all, the authors decided to include in the metaanalysis three papers [2-4] reporting a $0 \%$ specificity of chest CT in COVID-19 detection, whose design can easily explain this surprising data. As a matter of fact, all these studies enrolled only positive patients at RT-PCR, and therefore, the specificity could not be different from $0 \%$, because negative patients at RT-PCR were absent, and therefore, no patients could be classified as "true negative" by chest CT. Hence, we think that these studies should have been excluded from the meta-analysis, since their inclusion could have biased the final results on HRCT accuracy. Moreover,

Filippo Crimì

filippo.crimi@unipd.it

1 Institute of Radiology, Department of Medicine-DIMED,

University of Padova, Padova, Italy they may also have added a bias in the comparison of the pooled diagnostic accuracy among studies published by the Chinese and the Italian groups, since the sensitivity reported in the manuscripts was quite similar (76-100\% vs $91-97 \%)$, while the biggest differences were in the specificity $(0-96 \%$ vs $56-79 \%$ ).

Second, since a negative result of RT-PCR by nasopharyngeal swab does not rule out COVID-19 infection, especially in the mono-lateral involvement of lungs, as previously reported [5], the real specificity of the CT scan could be even higher than that reported by the studies included in the meta-analysis, because the presence of mono-lateral vs bilateral lung involvement at CT scan was not ever specified. We think that this observation could have added another bias and should have been reported among the study's limitations.

Finally, we cannot exclude an influence in the imaging accuracy by the different CT machines and scanning techniques employed in the studies included in the meta-analysis. As a matter of fact, an HRCT performed for the study of the lung parenchyma with a slice thickness of $1-1.5 \mathrm{~mm}$ can better detect subtle signs of early COVID-19 pulmonary infection (such as small ground glass opacities) than a normal CT scan with a slice thickness of $3 \mathrm{~mm}$ or larger. The majority of the studies employed thin slice thickness $(1-1.5 \mathrm{~mm}$ HRCT); however, a not irrelevant number of papers did not specify this technical data or reported a slice thickness of $3 \mathrm{~mm}$ or larger (5-7 mm). In our opinion, even this issue should have been appointed in the limitations section.

In summary, while we agree with the conclusions that chest CT can be a useful diagnostic tool for clinicians in the setting of the COVID-19 infection and that probably CT cannot be considered a "stand alone" technique for the diagnosis of the COVID-19 infection, it should be underlined that the multiple biases introduced in the meta-analysis affect the robustness of the final recommendation drawn by the authors. 


\section{Declarations}

Conflict of interest FC, GC, CZ and EQ have no conflicts of interest to declare.

Ethical approval This article does not contain any studies with human or animal subjects performed by any of the authors.

\section{References}

1. Pang C, Hou Q, Yang Z, Ren L (2021) Chest computed tomography as a primary tool in COVID-19 detection: an update metaanalysis. Clin Transl Imaging 26:1-11. https://doi.org/10.1007/ s40336-021-00434-Z

2. Xie X, Zhong Z, Zhao W, Zheng C, Wang F, Liu J (2020) Chest CT for typical coronavirus disease 2019 (COVID-19) pneumonia: relationship to negative RT-PCR testing. Radiology 296(2):E41E45. https://doi.org/10.1148/radiol.2020200343
3. Long C, Xu H, Shen Q, Zhang X, Fan B, Wang C, Zeng B, Li Z, Li X, Li H (2020) Diagnosis of the Coronavirus disease (COVID19): rRT-PCR or CT? Eur J Radiol 126:108961. https://doi.org/ 10.1016/j.ejrad.2020.108961 (Epub 2020 Mar 25)

4. Fang Y, Zhang H, Xie J, Lin M, Ying L, Pang P, Ji W (2020) Sensitivity of chest CT for COVID-19: comparison to RT-PCR. Radiology 296(2):E115-E117. https://doi.org/10.1148/radiol. 2020200432 (Epub 2020 Feb 19)

5. Quaia E, Baratella E, Crimi F, Cancian L, Crivelli P, Vianello A (2020) High-resolution CT features in patients with COVID19 pneumonia and negative nasopharyngeal and oropharyngeal swabs. Pulmonology. https://doi.org/10.1016/j.pulmoe.2020.10. 001 (Online ahead of print)

Publisher's Note Springer Nature remains neutral with regard to jurisdictional claims in published maps and institutional affiliations. 